\title{
Effects of the loss of estrogen on the heart's hypertrophic response to chronic left ventricle volume overload in rats
}

\author{
Elisabeth Walsh-Wilkinson ${ }^{1}$, Catherine Beaumont ${ }^{1}$, Marie-Claude Drolet ${ }^{1}$, Ève-Marie Roy ${ }^{1}$, Charlie Le Houillier ${ }^{1}$, \\ Jonathan Beaudoin $^{1}$, Marie Arsenault ${ }^{1}$, Jacques Couet ${ }^{\text {Corresp. } 1}$ \\ 1 Université Laval, Groupe de recherche sur les valvulopathies, Centre de recherche, Institut universitaire de cardiologie et de pneumologie de Québec, \\ Québec, Québec, Canada \\ Corresponding Author: Jacques Couet \\ Email address: jacques.couet@med.ulaval.ca
}

Aortic valve regurgitation (AR) can result in heart failure from chronic overloading of the left ventricle (LV). Little is known of the role of estrogens in the LV responses to this condition. The aim of the study was to compare LV remodeling in female rats with severe $A R$ in absence of estrogens by ovariectomy (Ovx). In a first study, we investigated over 6 months the development of hypertrophy in 4 groups of female Wistar rats: AR or shamoperated (sham) and Ovx or not. Ovx reduced normal heart growth. As expected, volume overload (VO) from AR resulted in significant LV dilation (42\% vs. 32\% increase LV enddiastolic diameter in intact and Ovx groups vs. their respective sham group; $p<0.0001$ ). LV weight was also significantly and similarly increased in both AR groups (non-Ovx and Ovx). Increase in stroke volume or cardiac output and loss of systolic function were similar between AR intact and AR Ovx groups compared to sham. We then investigated what were the effects of 17 beta-estradiol (E2; $0.03 \mathrm{mg} / \mathrm{kg} / \mathrm{day}$ ) treatment on the parameters studied in Ovx rats. Ovx reduced uterus weight by $85 \%$ and E2 treatment restored up to $65 \%$ of the normal weight. E2 also helped normalize heart size to normal values. On the other hand, it did not influence the extent of the hypertrophic response to AR. In fact, E2 treatment further reduced LV hypertrophy in AR Ovx rats (41\% over Sham Ovx + E2). Systolic and diastolic functions parameters in AR Ovx + E2 were similar to intact AR animals. Ovx in sham rats had a significant effect on the LV gene expression of several hypertrophy markers. Atrial natriuretic peptide (Nppa) gene expression was reduced by Ovx in sham-operated females whereas brain natriuretic peptide (Nppb) expression was increased. Alpha (Myh6) and beta (Myh7) myosin heavy chain genes were also significantly modulated by Ovx in sham females. In AR rats, LV expression of both Nppa and Nppb genes were increased as expected. Ovx further increased it of AR rats for Nppa and did the opposite for Nppb. Interestingly, AR in Ovx rats had only minimal effects on Myh6 and Myh7 genes whereas they were modulated as expected for intact AR animals. In summary, loss of estrogens bv Ovx in $\mathrm{AR}$ rats was not accompanied by a worsening of hypertrophy or
Peer) reviewing PDF (2019:06:38958:2:0: NEW 16 Sep 2019) 
cardiac function. Normal cardiac growth was reduced by Ovx in sham females but not the hyprtrophic response to AR. On the other hand, Ovx had important effects on LV gene expression both in sham and AR female rats. 


\title{
Effects of the loss of estrogen on the
}

2 heart's hypertrophic response to chronic left ventricle volume overload in rats.

\section{Élisabeth Walsh-Wilkinson, Catherine Beaumont, Marie-Claude Drolet, Ėve-Marie Roy, Charlie Le Houillier, Jonathan Beaudoin, Marie Arsenault and Jacques Couet. ${ }^{1}$}

\author{
${ }^{1}$ Groupe de recherche sur les valvulopathies, Centre de recherche, Institut universitaire \\ de cardiologie et de pneumologie de Québec, Université Laval, Québec, Québec, \\ Canada \\ Corresponding author: \\ Jacques Couet \\ Email address: jacques.couet@med.ulaval.ca
}

\begin{abstract}
Aortic valve regurgitation (AR) can result in heart failure from chronic overloading of the left ventricle (LV). Little is known of the role of estrogens in the LV responses to this condition. The aim of the study was to compare LV remodeling in female rats with severe AR in absence of estrogens by ovariectomy (Ovx). In a first study, we investigated over 6 months the development of hypertrophy in 4 groups of female Wistar rats: AR or sham-operated (sham) and Ovx or intact. Ovx reduced normal heart growth. As expected, volume overload (VO) from AR resulted in significant LV dilation ( $42 \%$ vs. $32 \%$ increase LV end-diastolic diameter in intact and Ovx groups vs. their respective sham group; $p<0.0001$ ). LV weight was also significantly and similarly increased in both AR groups (intact and Ovx). Increase in stroke volume or cardiac output and loss of systolic function were similar between AR intact and AR Ovx groups compared to sham. We then investigated what were the effects of 17beta-estradiol (E2; 0.03 $\mathrm{mg} / \mathrm{kg} /$ day) treatment on the parameters studied in Ovx rats. Ovx reduced uterus weight by $85 \%$ and E2 treatment restored up to $65 \%$ of the normal weight. E2 also helped normalize heart size to normal values. On the other hand, it did not influence the extent of the hypertrophic response to AR. In fact, E2 treatment further reduced LV hypertrophy in AR Ovx rats (41\% over Sham Ovx + E2). Systolic and diastolic functions parameters in AR Ovx + E2 were similar to intact AR animals. Ovx in sham rats had a significant effect on the LV gene expression of several hypertrophy markers. Atrial natriuretic peptide (Nppa) gene expression was reduced by Ovx in sham-operated females whereas brain natriuretic peptide $(\mathrm{Nppb})$ expression was increased. Alpha (Myh6) and beta (Myh7) myosin heavy chain genes were also significantly modulated by Ovx in sham females. In AR rats, LV expression of both Nppa and Nppb genes were increased as expected. Ovx further increased it of AR rats for Nppa and did the opposite for Nppb. Interestingly, AR in Ovx rats had only minimal effects on Myh6 and Myh7 genes whereas they were modulated as expected for intact AR animals. In summary, loss of estrogens by Ovx in AR rats was not accompanied by a worsening of hypertrophy or cardiac function. Normal cardiac growth was reduced by Ovx in sham females but not the hyprtrophic response to AR. On the other hand, Ovx had important effects on LV gene expression both in sham and AR female rats.
\end{abstract}

\section{INTRODUCTION}

Severe aortic valve regurgitation (AR) is a chronic disease that results in progressive left ventricular (LV) dilatation and eccentric hypertrophy. Although it is not the most frequent valvular disease in the Western world, it is estimated, based on the Framingham study, that $13 \%$ of the population suffers from some degree of AR. Singh et al. (1999) Moderate to severe secondary AR also occurs in a significant proportion of patients (5-10\%) undergoing transcatheter aortic valve replacement (TAVR). Leon et al. (2016) In poorer populations worldwide, mitral and/or aortic valve regurgitation is a frequent complication of acute rheumatic fever. Rheumatic valve diseases are still occurring at an alarming rate in low to 
middle-income countries and in poor communities, elsewhere.Zühlke et al. (2017) Gender differences in cardiac remodeling, hypertrophy and clinical outcome have been identified in various cardiac diseases such as heart failure, hypertension, aortic valve stenosis and experimental models of pressure and volume overload (VO). Blenck et al. (2016)Maric-Bilkan et al. (2016) The impact of valve regurgitation in women (mitral or aortic) has received little attention. Most clinical trials on chronic AR have focused mainly on male cohorts and gender specific adaptations of the LV in subjects suffering from chronic severe AR have not been investigated. Evangelista et al. (2005)Lin et al. (1994)Greenberg et al. (1988)

In prior studies using a rat model of chronic and severe AR, we showed that females had a hypertrophic response similar or stronger to LV VO than males. Drolet et al. (2006) Beaumont et al. (2017) The main difference was that the LV remodeling taking place in females was characterized by increased wall thickening resulting in a relatively preserved overall LV morphology compared to males (walls to chamber diameter ratio). Beaumont et al. (2017) Moreover, AR females showed a tendency for better survival than AR males. More recently, we observed that loss of androgens in male rats was associated with reduced cardiac growth and decreased LV hypertrophic response to severe AR. This suggests a role of male sex hormones in the control of both physiological and pathological cardiac growth and hypertrophy.Beaumont et al. (2019)

It is believed that estrogens can provide a protection against cardiac hypertrophy $(\mathrm{CH})$ development and evolution towards heart failure (HF). This has been demonstrated in animal models of pressure overload in female rats. Blenck et al. (2016) In the aorto-caval fistula (ACF) rat VO model, males progress faster toward HF and show poorer survival than females. Dent et al. (2010a) Since sex steroids have a potent effect on differentiation, they could thus explain a large part of the sex dimorphism observed in cardiac hypertrophy caused by VO. Leinwand (2003)

In the present study, we tested if LV remodeling and hypertrophy were influenced by estrogens in female rats suffering of chronic and severe AR. The effect of ovariectomy (Ovx) in females was assessed in order to answer this question. We also wanted to evaluate if Ovx was associated with decreased LV function and potentially worse survival in AR rats. In a second protocol, we studied the effects of 17beta-estradiol (E2) supplementation in AR Ovx female rats to investigate if it was associated with a reversal of the effects associated with the loss of estrogens.

\section{METHODS}

\section{Animals}

Fifty-eight female Wistar rats (225 to 250g) (Charles River, Saint-Constant, QC, Canada) were studied. Ovx females were purchased at the age of 9 weeks, one week after the procedure. Experimental groups were as followed: Sham-operated (Sham; $n=11)$, Ovx sham-operated (ShOvx; $n=10), A R ;(n=12)$ and Ovx AR (AROvx; $n=10)$. AR was induced at the age of 10 weeks as previously described by perforation of one or two aortic valve leaflets using a catheter via the right carotid and under echocardiographic guidance. Arsenault et al. (2002) Plante et al. (2003) Briefly, the right internal carotid artery was exposed and cannulated. Then, under continuous echocardiographic guidance, an 18-gauge epidural catheter was advanced toward the aortic valve in a retrograde manner. The sonographer guided the position and the advance of the catheter in the aorta while it was pushed through a leaflet of the aortic valve into the LV. Leaflet perforation was repeated if the severity of the regurgitant jet was considered insufficient by echocardiographic criteria. Sham-operated animals only had the ligation of their right carotid. Duration of the protocol was 6 months. Two additional Ovx groups were also studied, namely ShOvx + E2 (n=6) and AROvx + E2 ( $n=8)$. E2 (17beta-estradiol) was administered using a subcutaneous pellet implanted in the neck of the animal liberating $0.03 \mathrm{mg} / \mathrm{kg} / \mathrm{day}$ for 3 months (Innovative research of America, Sarasota, FL). After this period, a second pellet was implanted to complete the protocol. The protocol was approved by the Université Laval's Animal Protection Committee and followed the recommendations of the Canadian Council on Laboratory Animal Care.

\section{Echocardiography}

An echocardiographic exam (Philips HD11XE using an $12 \mathrm{MHz}$ probe (S12)) was performed under isoflurane anesthesia (2\%) two weeks after surgery to confirm AR severity and at the end of the protocol 26 weeks later, as previously described. Arsenault et al. (2013) Arsenault et al. (2002) Plante et al. (2003) The regurgitant fraction was estimated by the ratio of the forward systolic flow time-velocity integral (VTI) to the reversed diastolic flow VTI measured by pulsed Doppler in the thoracic descending aorta. 
Table 1. Name and symbol of all primer pairs used for gene expression analysis by quantitative RT-PCR. The table also includes catalogue numbers (from IDT or Qiagen) and the size of the amplicon.

\begin{tabular}{l|c|c|c} 
mRNA & Symbol & Catalog no. & Amplicon (bp) \\
\hline acyl CoA déshydrogenase, very long chain & Acadvl & Rn.PT.58.13279450 & 147 \\
carnitine palmitoyltransferase 2 & Cpt2 & QT00186473 & 150 \\
connective tissue growth factor & Ctgf & QT00182021 & 102 \\
cyclophilin A & Ppia & Rn.PT.39a,22214830 & 140 \\
cytochrome b-245 heavy chain (NOX2) & Nox2 & Rn.PT.58.17749203 & 97 \\
2,4-dienoyl CoA reductase 1 & Decr1 & Rn.PT.58.44352482 & 120 \\
enolase 3, beta & Eno3 & QT00180138 & 106 \\
estrogen related receptor, alpha & Erra & Rn.PT.58.5170310 & 111 \\
estrogen related receptor, gamma & Errg & Rn.PT.58.8028733 & 141 \\
fatty acid translocase/CD36 & Fat/CD36 & QT01702680 & 81 \\
hexokinase 1 & Hk1 & Rn.PT.58.8913174 & 108 \\
hydroxyacyl-CoA dehydrogenase & Hadh & Rn.PT.58.17867024 & 135 \\
hydroxyacyl-CoA dehydrogenase alpha & Hadha & Rn.PT.58.46222281 & 138 \\
lysyl oxidase, cardiac & Lox & Rn.PT.58.10677971 & 150 \\
myosin, heavy polypeptide 6, cardiac & Myh6 & Rn.PT.58.8646063 & 150 \\
myosin, heavy polypeptide 7, cardiac & Myh7 & Rn.PT.58.34623828 & 125 \\
NADPH oxidase 4 & Nox4 & Rn.PT.58.11992143 & 107 \\
natriuretic peptide precursor type A & Nppa & Rn.PT.58.5865224 & 79 \\
natriuretic peptide precursor type B & Nppb & Rn.PT.58.5595685 & 108 \\
phosphofructokinase & Pfkm & Rn.PT.58.17873275 & 122 \\
procollagen-1 alpha-1 & Col1 & Rn.PT.58.7562513 & 134 \\
procollagen-3 alpha-1 & Col3 & Rn.PT.58.11138874 & 100 \\
pyruvate dehydrogenase alpha 1 & Pdha1 & QT01830220 & 93 \\
pyruvate dehydrogenase kinase, isozyme 4 & Pdk4 & QT00189287 & 145 \\
retinoid X receptor gamma & Rxrg & Rn.PT.58.6519292 & 103 \\
solute carrier family 2 member 1 & Glut1 & QT00178024 & 85 \\
solute carrier family 2 member 4 & Glut4 & QT00175931 & 146 \\
superoxide dismutase 1, soluble & SOD1 & Rn.PT.58,5432362 & 138 \\
superoxide dismutase 2, mitochondrial & SOD2 & Rn.PT.58.7509049 & 107 \\
superoxide dismutase 3, extracellular & SOD3 & QT00379358 & 92 \\
\hline
\end{tabular}

At the end of the protocol, the heart and the lungs were harvested and weighed. Heart chambers were dissected, weighted and the LV was then quickly frozen in liquid nitrogen and kept at $-80 \mathrm{C}$ until further use.

\section{Gene Expression Analysis by quantative RT-PCR}

LV gene expression was quantified for 6 animals per group by quantitative RT-PCR as described elsewhere. Champetier et al. (2009) Pre-optimized primers were from QuantiTect (Qiagen) and IDT (Coralville, Iowa) (Table 1) and SsoAdvanced Universal SYBR Green Supermix (Bio Rad, Hercules, CA) was used. We used one pair of non-pre-optimized primers for the enoyl CoA hydratase, short chain 1 gene (EchsI) (5'-GCTTTCAGGGTGTCTTGATTTG-3' and 5'-GAGCTATGCACTGCAGATAGT-3'; 95 bp transcript). We tested three different genes as possible housekeeping gene as control for this study. Cyclophilin A gene was chosen since it had the one most stable expression among the different groups.

\section{Statistical analysis}

Results are presented as the mean and the standard error of the mean (SEM). Two-way ANOVA analysis was performed and Holm-Sidak's post-test was used for comparison between the groups (Graph Pad Prism 8.1, San Diego, CA). A Student's t-test was used when only two groups were compared. A p-value lower than 0.05 was considered significant. 


\section{RESULTS}

\section{Effects of overiectomy on the hypertrophic response to chronic volume overload}

AR was surgically induced in intact (non-OVX) and Ovx Wistar female rats at the age of 10 weeks. The protocol had a duration of 26 weeks (6 months). All animals survived the duration of the protocol. In Table 2 are summarized the characteristics of the animals at the end of the protocol. Sham Ovx females were smaller and their heart lighter compared to Sham. Indexed heart weight for tibial length was also lower for Sham Ovx compared to Sham. When indexed for body weight, no difference was present. As expected, AR caused important increases in total heart weight as well as for the left ventricle and left atria. This increase was similar for both intact (non-Ovx) and Ovx animals (75\% vs. $70 \%)$ as illustrated in Figure 1. In order to confirm that Ovx resulted in a loss of sex hormones, we weighed the uterus, a tissue strongly dependant on estrogens. As expected, uterine weight was markedly decreased (84\%) in both Ovx groups (sham and AR).

Table 2. Characteristics of the animals at the end of the protocol. BW: body weight. Values are expressed as the mean +/- SEM. Group comparisons were made using two-way ANOVA analysis and Holm-Sidak's post-test. *: $\mathrm{p}<0.001$ vs the respective sham group and $\S: \mathrm{p}<0.05$ vs non-Ovx group.

\begin{tabular}{l|c|c|c|c} 
Parameters & $\begin{array}{c}\text { Sham } \\
(\mathrm{n}=11)\end{array}$ & $\begin{array}{c}\text { AR } \\
(\mathrm{n}=13)\end{array}$ & $\begin{array}{c}\text { Sham Ovx } \\
(\mathrm{n}=10)\end{array}$ & $\begin{array}{c}\text { AR Ovx } \\
(\mathrm{n}=10)\end{array}$ \\
\hline Body weight, g & $428+/-15$ & $418+/-20$ & $368+/-11 \S$ & $417+/-14^{*}$ \\
Tibia, mm & $51+/-0.2$ & $53+/-0.3^{*}$ & $50+/-0.3 \S$ & $50+/-0.2 \S$ \\
Heart, mg & $963+/-20$ & $1685+/-31^{*}$ & $765+/-20 \S$ & $1304+/-25^{*} \S$ \\
Heart/BW, mg/g & $2.3+/-0.1$ & $4.1+/-0.2^{*}$ & $2.1+/-0.1$ & $3.2+/-0.1^{*} \S$ \\
Heart/TL, mg/mm & $18.8+/-0.3$ & $31.9+/-0.6^{*}$ & $15.4+/-0.4 \S$ & $26.0+/-0.5^{*} \S$ \\
Left ventricle, mg & $735+/-17$ & $1354+/-25^{*}$ & $588+/-13 \S$ & $1015+/-25^{*} \S$ \\
Left atria, mg & $25+/-3$ & $47+/-3^{*}$ & $18+/-2$ & $32+/-2^{*}$ \\
Lungs, g & $1.7+/-0.1$ & $3.2+/-0.3^{*}$ & $2.4+/-0.2$ & $2.2+/-0.3$ \\
Uterus, mg & $59+/-4$ & $59+/-3$ & $9+/-1 \S$ & $9+/-1 \S$ \\
\hline
\end{tabular}

As for the animal and heart characteristics described above, most echocardiographic parameters were significantly changed by AR (Table 3). AR severity was similar between both AR groups. LV end-diastolic diameter was smaller in AROvx animals compared to AR group. This was also the case for the stroke volume (SV) and the cardiac output (CO). Ejection fraction (EF; an index of systolic function) was reduced in both AR groups. Interestingly, loss of estrogens also associated with a reduced EF in Sham Ovx animals. The E wave, representing LV passive filling was significantly increased in AR animals compared to AR Ovx ones.

In Figure 1, we illustrated variations of several parameters mentioned above in AR animals relative to their respective sham-operated group. As expected, AR caused important cardiac hypertrophy in both AR and AROvx animals compared to sham and this increase in heart weight was similar for both groups. A tendency for a greater increase in LV weight and LV end-diastolic diameter (EDD) caused by AR was recorded for the AR group compared to AROvx but this did not reach statistical significance (Figure 1 $\mathrm{B}-\mathrm{C})$. Ejection fraction (EF), LV stroke volume (SV) and cardiac output (CO) were all modified by VO from AR but again, there was no difference from the hormonal status (AR group vs. AROvx) (Figure 1D-F).

\section{Effects of E2 treatment on cardiac hypertrophy in AR Ovx females}

We then studied the effects of E2 treatment in both Sham Ovx (ShOvx) and AR Ovx (ArOvx) rats. As summarized in Table 4, Ovx rats treated with E2 were still smaller than non-Ovx ones (see Table 2 for comparison). On the other hand, indexed heart weight was normalized suggesting that cardiac growth was not slowed in ShOvx $+\mathrm{E} 2$ rats. AR produced heart and LV hypertrophy but relatively less than for sham and Ovx animals (around 40\% increase in AROvx + E2 compared to 70\% for untreated AROvx; Table 2). Uterine weight was increased by E2 treatment to approximately $65 \%$ of these of non-Ovx females.

As illustrated in Figure 2, E2 treatment partly normalized cardiac growth in Sham Ovx females. Heart and LV weights were also significantly increased in Sham Ovx rats receiving E2 compared to those untreated. Moreover, LV stroke volume (SV) and cardiac output (CO were completely normalized by E2 
Table 3. Echocardiographic parameters of sham-operated animals at the end of the protocol. EDD: end-diastolic diameter, ESD: end-systolic diameter, SW: septum wall thickness, PW: posterior wall thickness, EF: ejection fraction, RWT: relative wall thickness, SV: stroke volume, HR: heart rate, bpm: beats per minute, CO: cardiac output and na: non applicable. Values are expressed as the mean +/- SEM. Group comparisons were made using two-way ANOVA analysis and Holm-Sidak's post-test. *:p $<0.05$ vs. respective sham group and $\S: \mathrm{p}<0.05$ vs. non-Ovx group.

\begin{tabular}{l|c|c|c|c} 
Parameters & $\begin{array}{c}\text { Sham } \\
(\mathrm{n}=11)\end{array}$ & $\begin{array}{c}\text { AR } \\
(\mathrm{n}=13)\end{array}$ & $\begin{array}{c}\text { Sham Ovx } \\
(\mathrm{n}=10)\end{array}$ & $\begin{array}{c}\text { AR Ovx } \\
(\mathrm{n}=10)\end{array}$ \\
\hline AR severity, \% & $\mathrm{na}$ & $83+/-4$ & $\mathrm{na}$ & $78+/-2$ \\
\hline EDD, mm & $7.7+/-0.1$ & $10.9+/-0.2$ & $7.4+/-0.1$ & $9.8+/-0.2^{*} \S$ \\
ESD, mm & $3.1+/-0.1$ & $6.5+/-0.3^{*}$ & $3.6+/-0.1$ & $6.2+/-0.3^{*}$ \\
$\mathrm{SW}, \mathrm{mm}$ & $1.1+/-0.02$ & $1.4+/-0.05^{*}$ & $1.2+/-0.04$ & $1.4+/-0.04^{*}$ \\
PW, mm & $1.2+/-0.03$ & $1.8+/-0.08^{*}$ & $1.4+/-0.07$ & $1.5+/-0.06^{*}$ \\
EF, \% & $84+/-2$ & $65+/-2^{*}$ & $75+/-2 \S$ & $61+/-3^{*}$ \\
RWT, unitless & $0.28+/-0.005$ & $0.26+/-0.011$ & $0.29+/-0.010$ & $0.27+/-0.007$ \\
SV, ml & $0.29+/-0.01$ & $0.52+/-0.04^{*}$ & $0.22+/-0.01 \S$ & $0.40+/-0.02^{*} \S$ \\
HR, bpm & $386+/-13$ & $379+/-9$ & $348+/-16$ & $373+/-11$ \\
CO, ml/min & $113+/-3$ & $187+/-10^{*}$ & $79+/-7 \S$ & $148+/-8^{*} \S$ \\
E wave, cm/s & $95+/-4$ & $109+/-4$ & $83+/-4$ & $86+/-3 \S$ \\
A wave, cm/s & $61+/-3$ & $57+/-2$ & $59+/-7$ & $47+/-2$ \\
E wave slope & $2992+/-199$ & $3379+/-305$ & $2098+/-215 \S$ & $2850+/-135$ \\
\hline
\end{tabular}

Table 4. Animal characteristics of Ovx animals treated with 17beta-estradiol (E2) at the end of the protocol. BW: body weight. Values are expressed as the mean +/- SEM. Group comparisons were made using Student's T-test.

\begin{tabular}{l|c|c|c} 
Parameters & $\begin{array}{c}\text { ShOvx }+ \text { E2 } \\
(\mathrm{n}=6)\end{array}$ & $\begin{array}{c}\text { AROvx + E2 } \\
(\mathrm{n}=8)\end{array}$ & $\mathrm{p}$-value \\
\hline Body weight, g & $342+/-8$ & $320+/-11$ & 0.096 \\
Tibial length, mm & $48+/-0.3$ & $48+/-0.3$ & 0.69 \\
Heart, mg & $870+/-26$ & $1223+/-39$ & $<0.0001$ \\
Heart/BW, mg/g & $2.5+/-0.07$ & $3.9+/-0.10$ & $<0.0001$ \\
Heart/TL, mg/mm & $18.0+/-0.5$ & $25.6+/-0.7$ & $<0.0001$ \\
Left ventricle, mg & $650+/-20$ & $973+/-24$ & $<0.0001$ \\
Left atria, mg & $21+/-1$ & $34+/-3$ & $<0.0001$ \\
Lungs, mg & $1.5+/-0.1$ & $1.6+/-0.1$ & 0.89 \\
Uterus, mg & $37+/-2.3$ & $40+/-3.1$ & 0.55 \\
\hline
\end{tabular}

treatment in sham females (Table 5 and Figure $2 \mathrm{E}$ and $2 \mathrm{~F}$ ). Systolic function as evaluated by ejection fraction (EF) was unchanged be E2 treatment Figure 2D.

\section{LV gene expression modulation by estrogens}

We then measured LV gene expression for several hypertrophy markers. Atrial natriuretic peptide (Nppa or Anp) and brain natriuretic (Nppb or Bnp) mRNA levels were both modulated by the loss of estrogens in Sham Ovx animals (Figure 3A). Nppa levels were reduced by $60 \%$ whereas $N p p b$ levels remained stable. Ovx modulated myosin heavy chain gene expression in a similar fashion as often observed in cardiac hypertrophy. Myosin heavy chain alpha (Myh6) gene expression was reduced by Ovx whereas myosin heavy chain beta $(M y h 7)$ was increased compared to non-Ovx Sham animals. Nppa mRNA levels were strongly increased in AR animals compared to corresponding Sham group; this raise being stronger in the AROvx group (Figure 3B). We observed the opposite trend for Nppb mRNA levels, which were more strongly increased in AR females than in AROvx ones. A similar situation was observed for the expression of myosin heavy chain genes in AR rats. Myh6 gene expression was reduced Myh7 increased by AR. Those modulations were less important in the AR Ovx group. Loss of estrogens lead to a decrease in procollagen 1 (Collal) and procollagen 3 (Col3) gene expression in sham-operated rats (Figure 3C). 

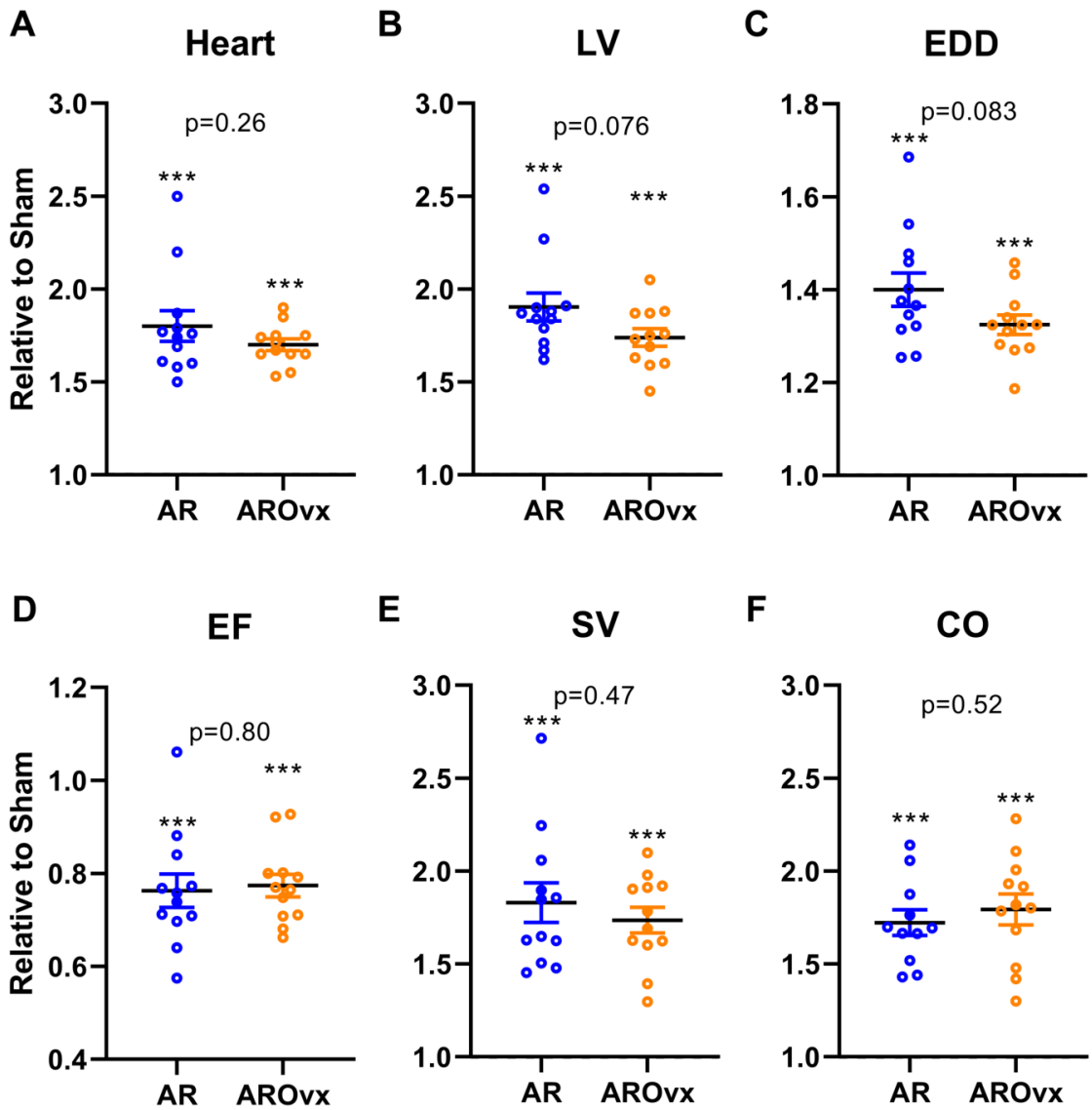

Figure 1. Ovariectomy does not modulate the hypertrophic response triggered by AR. Results are expressed in arbitrary units (mean +/- SEM) relative to their respective sham-operated group (fixed to 1). A: Heart, B: LV, Left ventricular weight, C: EDD, end-diastolic diameter, D: EF, ejection fraction, E: SV, stroke volume and F: CO, cardiac output. Calculated p values (Student's T-test) are indicated for comparison between AR and AROvx groups. ***: $\mathrm{p}<0.001$ compared to respective sham group (non-Ovx or Ovx).

Collal and Col3 mRNA levels remained normal in AR animals (Figure 3D) but were slightly more elevated in AROvx animals. The same was observed of mRNA levels of lysyl oxidase 1 gene $(L o x)$ in AR groups. CTGF gene expression levels were unchanged by Ovx and were significantly increased by AR (Figure 3C-D). We then tested the expression of genes encoding transcription factors implicated in the control of myocardial energetics (Figure 3E). Estrogen-related receptors (alpha and gamma) and retinoic $\mathrm{X}$ receptor gamma mRNA levels were measured in the $\mathrm{LV}$ of the animals. ERR alpha levels were reduced by Ovx in sham animals but not further by AR. Moreover, mRNA levels of these three transcription factor genes were significantly decreased by AR but loss of estrogens restored these levels to normal (Figure $3 \mathrm{~F})$.

We reported previously that female AR rats unlike males, kept a relatively normal transcriptional profile of many genes related to myocardial energetics. Beaumont et al. (2017) Here, we were interested to see if loss of estrogens would induce pertubation to this. We thus tested a number of genes related to fatty acids beta-oxidation and glycolysis. In addition, we measured LV mRNA levels of various genes associated to reactive oxygen species (ROS) metabolism. As illustrated in Figure 4, loss of estrogens via Ovx had very little effects on LV expression of various genes implicated in myocardial energetics except for one, Pdk4 (Figure 4A and B). AR reduced the expression of a number of genes namely Acadvl, Decrl, Hadh, HadhA, Eno3 and Pdk4. Loss of estrogens did not further modulated those genes (AROvx rats). 

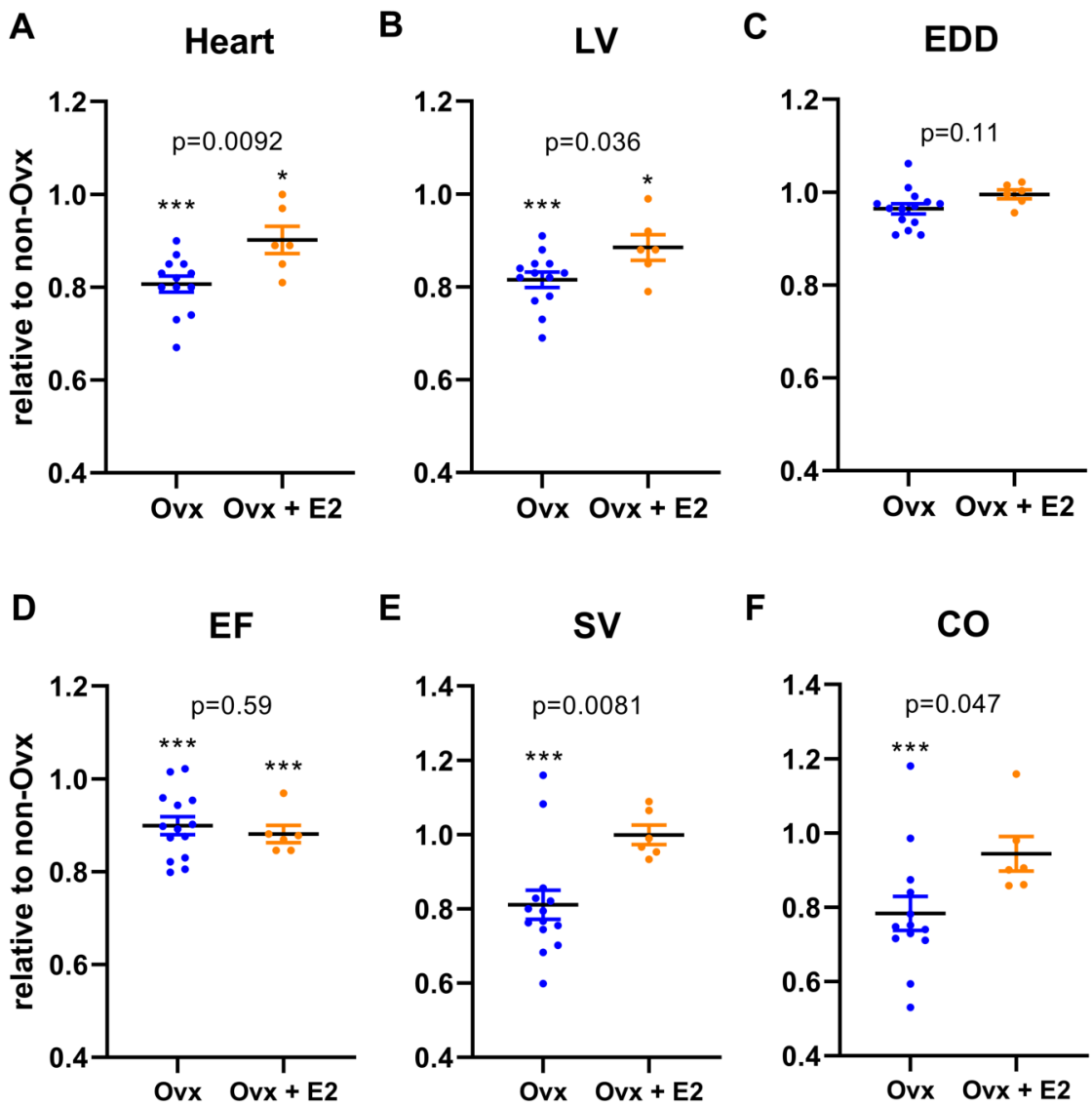

Figure 2. Ovariectomy (Ovx) slows normal heart growth in Wistar female rats and $\mathrm{E} 2$ treatment partially reverses this effect. Results are expressed as the ratio of the indicated parameter (mean +/- SEM) compared to the mean of the same parameter for non-Ovx Sham females (set to 1). A: Heart, B: LV, Left ventricular weight, C: EDD, end-diastolic diameter, D: EF, ejection fraction, E: SV, stroke volume and F: $\mathrm{CO}$, cardiac output. Calculated $\mathrm{p}$ values (Student's T-test) are indicated for comparison between Sham $(\mathrm{Ovx})$ and Sham $(\mathrm{Ovx}+\mathrm{E} 2)$ groups. *: $\mathrm{p}<0.05$ and $* * *: \mathrm{p}<0.001$ compared to non-Ovx sham female group.

Among the genes related to ROS metabolism, we observed that NADPH oxidase 4 (Nox4) expression was significantly reduced by Ovx. This was reversed by AR. AR up-regulated Nox2 in both intact and Ovx females.

We then studied if E2 treatment of Ovx animals helped restored changes observed in natriuretic peptides (Nppb or Bnp) and myosin heavy chains (Myh6 and Myh7) gene expression. Interestingly, E2 helped normalize Nppa and Myh7 expression in Sham animals (Figure 5). Decreased Myh6 gene expression in Sham Ovx females was not normalized by E2, however. E2 treatment had no effect on gene expression levels in AR animals.

\section{DISCUSSION}

In this study, we observed that loss of estrogens by ovariectomy (Ovx) two weeks before AR induction had relatively little effects on the extent of the cardiac response to a LV VO, at least at the macroscopic level. Ovx resulted in slower cardiac growth in Sham female rats during the 6 months that lasted the protocol. This was partly reversed by 17 beta-estradiol treatment. On the other hand, LV hypertrophy caused by severe VO from AR was similar in all AR groups, non-Ovx, Ovx and Ovx receiving E2. We had previously shown that LV remodeling from AR in this model involved similar LV dilation in rats of 
Table 5. Echocardiographic parameters of of Ovx animals treated with 17beta-estradiol (E2) at the end of the protocol. EDD: end-diastolic diameter, ESD: end-systolic diameter, SW: septum wall thickness, PW: posterior wall thickness, EF: ejection fraction, RWT: relative wall thickness, SV: stroke volume, HR: heart rate, bpm: beats per minute, $\mathrm{CO}$ : cardiac output and na: non applicable. Values are expressed as the mean +/- SEM. Group comparisons were made using Student's T-test.

\begin{tabular}{l|c|c|c} 
Parameters & $\begin{array}{c}\text { ShOvx + E2 } \\
(\mathrm{n}=6)\end{array}$ & $\begin{array}{c}\text { AROvx }+ \text { E2 } \\
(\mathrm{n}=8)\end{array}$ & p-value \\
\hline AR severity, \% & na & $66+/-2$ & na \\
\hline EDD, mm & $7.7+/-0.1$ & $9.7+/-0.1$ & $<0.0001$ \\
ESD, mm & $3.9+/-0.1$ & $5.6+/-0.3$ & $<0.0001$ \\
SW, mm & $1.1+/-0.03$ & $1.3+/-0.05$ & 0.0019 \\
PW, mm & $1.3+/-0.02$ & $1.4+/-0.10$ & $<0.0001$ \\
EF, \% & $74+/-2$ & $67+/-3$ & 0.073 \\
RWT, unitless & $0.27+/-0.007$ & $0.26+/-0.010$ & 0.55 \\
SV, ml & $0.30+/-0.01$ & $0.44+/-0.02$ & $<0.0001$ \\
HR, bpm & $362+/-16$ & $351+/-10$ & 0.56 \\
CO, ml/min & $107+/-5$ & $153+/-8$ & 0.0005 \\
E wave, cm/s & $90+/-3$ & $111+/-5$ & 0.0024 \\
A wave, cm/s & $55+/-2$ & $67+/-6$ & 0.051 \\
E wave slope & $2700+/-187$ & $3363+/-255$ & 0.0033 \\
\hline
\end{tabular}

both sexes but more wall thickening in females. This resulted in AR females in maintained LV relative wall thickness (RWT) although significant hypertrophy was present.Beaumont et al. (2017) RWT also remained stable in sham-operated females after Ovx as well as in all AR groups. Our results suggest that loss of estrogens seems to clearly influence more cardiac normal growth than the response to chronic and severe VO in the AR rat model.

The roles of estrogens in pathological cardiac hypertrophy has been studied mostly in pressure overload animal models. It received less attention in VO situations such as in valve regurgitation models or in the aortocaval fistula (ACF) model. ACF is a model of global cardiac VO model. It is less relevant from a clinical standpoint but it remains the most studied pre-clinical VO model. Female ACF rats were shown to develop less hypertrophy, to evolve more slowly towards heart failure and to display better overall survival than males. Gardner et al. (2002) This advantage over males was dependant on estrogens as ovariectomy reversed these benefits. Brower et al. (2003) Dent and collaborators characterized this ACF model further and showed that 17beta-estradiol could help normalize the effects of ovariectomy. Dent et al. (2010b) Some discrepencies seem to exist between findings described in the present study in AR rats and those reported previously in the ACF model. A few differences have to be highlighted between these models. In the ACF model studies, evolution towards heart failure was documented (at least in males) whereas in the AR model, overt heart failure symptoms are a rare occurrence. In fact, most of the deaths are sudden happening during the active period of the animals during the night. Arsenault et al. (2013)Lachance et al. (2009)Plante et al. (2008). Since ACF is a global form of VO targeting the right heart first, it is likely that than lungs become seriously affected sooner, which leads to heart failure.

More than a decade ago, we had reported that Ovx was not associated with major effects in AR females. Drolet et al. (2006) More recently, we showed that LV dilation caused by AR had similarities between males and female rats, but that the expression profile of many genes involved in myocardial energetics was strongly modulated in males but not in females. Beaumont et al. (2017) This suggested that AR females could probably keep a relatively normal myocardial energy metabolism or at least, a better energy substrate use flexibility even in a situation of pathological hypertrophy. In addition, myocardial capillaries density in AR females was not decreased as in males suggesting better oxygen and nutrients availability for surrounding cardiac myocytes. Removing androgens by orchiectomy (Ocx) in AR males reversed some of these sex differences. As observed in the present study for females, normal cardiac growth in male rats was also dependant on the presence of sex hormones. Unlike for Ovx females, hypertrophic response to severe VO was clearly decreased in Ocx AR males. Beaumont et al. (2019) Here, we observed that estrogens had minimal effects if any, on the LV remodeling taking place after AR induction. LV dilation, increase in stroke volume and cardiac output were similar in both non-Ovx and 
Ovx AR groups. This suggests that the hypertrophic response to a similar and direct LV pathological stress such as with AR required a similar myocardial adaptations to accommodate the additional regurgitating blood to pump. This was not influence by the hormonal status. Moreover, this observation suggests the female sex irrespective of estrogens can provide benefits in this rat model. It is also possible that imprinting of estrogens from the early life of the animals still remains.

The steroid hormone 17 beta-estradiol is a key player in many biological processes, such as reproduction, development, metabolism, cell proliferation and differentiation. Deroo and Korach (2006) Estrogens are implicated in the regulation of many genes and signaling pathways via genomic and non-genomic actions. E2 can bind and mediate its actions via the estrogen receptors (ER) ERalpha and ERbeta, which can then act as transcription factors for specific sets of genes. Membrane-associated receptor such as GPER and ERs, can also be activated by E2, resulting in the modulation of cytoplasmic signalling cascades and ultimately regulations of target genes. Murphy (2011) Post-natal heart growth occurs via cardiomyocytes hypertrophy since these cells are post-mitotic. Activation of the ERalpha is required for post-natal heart growth in healthy Ovx mice (C57B16/J strain) receiving E2 suggesting a central role for this receptor.Kararigas et al. (2014) ERbeta does not seem to be involved in normal heart growth in female mice but is believed to be implicated in the protection of the heart during a pathological stress.Mahmoodzadeh and Dworatzek (2019) Estrogens can produce effects on the heart of males and females since ERs are present in the myocardium of both sexes. Estrogens as a potential therapy in men with cardiac diseases have received less attention than for women. E2 has been shown to rescue male mice with heart failure from transverse aortic constriction (a pressure overload model) via in part the ERbeta receptor and GPER. Iorga et al. (2016)Iorga et al. (2018) In the ACF model, estrogen therapy in males was able to reduce the hypertrophic response to the volume overload. Gardner et al. (2009) It would be interesting in the future, to investigate E2 effects in male AR animals in order to know if their effect could be more beneficial than in females.

Pathological cardiac hypertrophy is associated with an important remodelling of the myocardial structure, a consequence of cardiomyocyte size increase and extracellular matrix rearrangement. Neurohormonal factors as well as mechanic stress cause alterations in myocardial gene expression including the reactivation of the fetal gene program. Taegtmeyer et al. (2010) This feature is common to a variety of pathological conditions including ischemia, atrophy, hypoxia, diabetes in addition of hypertrophy. This return to the fetal gene program has long been considered detrimental, whereas others have suggested that it protects the heart against irreversible impairment and cell death. Genes associated with the fetal gene program include atrial and brain natriuretic peptide ( $N p p a$ and $N p p b)$, contractile protein beta-myosin heavy chain (beta-MHC or $M y h 7$ ) and early response genes such as $c$-myc and $c$-fos among many others. This reactivation of the fetal gene program in the stressed heart is accompanied with the down-regulation of the adult gene program. Rajabi et al. (2007) Here, we observed that Ovx was associated with the modulation of several genes associated with the fetal program in female rats. LV atrial natriuretic peptide (Nppa) and contractile protein beta-myosin heavy chain (Myh6 and $M y h 7)$ were all significantly modulated by the loss of estrogens. This was also true for other genes often modulated in cardiac hypertrophy such as collagen genes (Collal and Col3), ERRalpha, Pdh4) and Nox4. This modulation was not associated directly with an inhibition or a reactivation of the fetal gene program. For instance, Myh6 and Myh7 LV genes in ShamOvx rats followed the usual pattern associated with pathological hypertrophy. On the other hand, Nppa was down-regulated and Nppb expression remained unchanged. If Nppa and Myh7 gene expression was normalized by E2, it was not the case for Myh6.

We reported previously that genes associated with myocardial energetics were strongly modulated in AR male rats and that a clear sex dimorphism was present when compared to females. Arsenault et al. (2013)Beaumont et al. (2017) Pathological LV hypertrophy is usually associated with a shift towards glucose use and glycolysis instead of the preferred beta-oxidation of fatty acids (FAO). We observed this shift in our model in males and this correlated with down-regulation of many genes involved in FAO.Arsenault et al. (2013)Lachance et al. (2014) Although, several FAO genes were downregulated mildly in female AR rats compared to normal controls, the overall transcriptional profile remained near normal suggesting that they probably maintained a better energy substrates flexibility than males.Beaumont et al. (2017) Loss of androgens in males helps normalize this general down-regulation of FAO genes.Beaumont et al. (2019) We were thus interested to see if loss of estrogens would impact negatively AR females, which was not case as observed here. This suggest that androgens are probably more implicated in this control of myocardial energetics in pathological hypertrophy. As mentioned, the 
better angiogenic response during myocardial remodeling in females is probably a important contributing factor to the maintenance of relatively normal energetics.

The results obtained in the present study on female AR rats in conjunction to those we recently reported in males demonstrate that sex hormones are not the sole factors intervening in the LV hypertrophic response. Beaumont et al. (2019) Both androgens and estrogens are important for normal cardiac growth. Loss of estrogens by Ovx slows down cardiac growth and E2 treatment helps reverse this effect. Levels of LV hypertrophy are equivalent between AR males and females. Loss of testosterone reduces the extent of LV hypertrophy in AR rats whereas loss of E2 has relatively little effects. In addition, Ovx in AR rats is not associated with a worse transcriptional profile of genes normally regulated in cardiac hypertrophy. In fact, expression of several hypertrophy markers such as myosin heavy chain genes (Myh6 and Myh7) was in part normalized by Ovx in AR females as well as for mRNA levels of Nppb, Err alpha and Err gamma. Again, loss of androgens seemed to provide some benefits to males on this aspect whereas estrogens are mainly neutral in females. Beaumont et al. $(2017,2019)$ In summary, we did not identify clear negative impact of the loss of estrogens in AR female rats in a chronic setting. Sexual dimorphism in the response to VO seems to rely more on the effects of androgens in males. It is also possible that the influence of sex hormones before gonadectomy is still imprinted later in the life of the animals. Finally, effects of sex chromosomes and the genes they harbor, should not be excluded.

We want to point out several limitations in this study. In the second part of the study where Ovx rats received E2, estimated severity of AR was less severe (66\% in AR Ovx + E2 vs. around $80 \%$ for AR and AR Ovx groups) and so was the hypertrophy relative to the respective sham group (41\% in AR Ovx + E2 vs. around $70-75 \%$ for AR and AR Ovx groups). On the other hand, if one considers the indexed heart weight gain between AR Ovx rats receiving or not E2, the hypertrophic response was similar. We did not measure circulating E2 levels and we used uterine weight as a surrogate of estrogen action. The dosage of E2 used reversed about two thirds of the expected uterus weight suggesting that it was probably a little low or that type of delivery could not reproduce the natural situation. This could explain in part, why cardiac growth was not restored to normal levels. Obviously, continuous release of E2 does not reproduce naturally occurring circadian rhythm of production and release of sex hormones in the body. In addition, E2 treatment alone cannot restore completely other possible hormonal imbalances created by Ovx. They too, may probably contribute to observations made in this study. Finally, Ovx was performed in young animals, which does not translate well to the situation of post-menopausal and older patients.

\section{CONCLUSION}

In conclusion, we showed that loss of estrogens was not associated with important effects on the hypertrophic response to severe and chronic aortic valve regurgitation in female Wistar rats.

\section{REFERENCES}

Arsenault, M., Plante, E., Drolet, M., and Couet, J. (2002). Experimental aortic regurgitation in rats under echocardiographic guidance. The Journal of heart valve disease, 11(1):128-134.

Arsenault, M., Zendaoui, A., Roussel, É., Drolet, M.-C., Dhahri, W., Grenier, A., Gascon, S., Sarrhini, O., Rousseau, J. A., Lecomte, R., and Couet, J. (2013). Angiotensin ii-converting enzyme inhibition improves survival, ventricular remodeling, and myocardial energetics in experimental aortic regurgitationclinical perspective. Circulation: Heart Failure, 6(5):1021-1028.

Beaumont, C., Walsh-Wilkinson, É., Drolet, M.-C., Roussel, É., Arsenault, M., and Couet, J. (2017). Female rats with severe left ventricle volume overload exhibit more cardiac hypertrophy but fewer myocardial transcriptional changes than males. Scientific reports, 7(1):729.

Beaumont, C., Walsh-Wilkinson, É., Drolet, M.-C., Roussel, É., Melançon, N., Fortier, É., Harpin, G., Beaudoin, J., Arsenault, M., and Couet, J. (2019). Testosterone deficiency reduces cardiac hypertrophy in a rat model of severe volume overload. Physiological reports, 7(9):e14088.

Blenck, C. L., Harvey, P. A., Reckelhoff, J. F., and Leinwand, L. A. (2016). The importance of biological sex and estrogen in rodent models of cardiovascular health and disease. Circ Res, 118(8):1294-312.

Brower, G. L., Gardner, J. D., and Janicki, J. S. (2003). Gender mediated cardiac protection from adverse ventricular remodeling is abolished by ovariectomy. Mol Cell Biochem, 251(1-2):89-95.

Champetier, S., Bojmehrani, A., Beaudoin, J., Lachance, D., Plante, É., Roussel, E., Couet, J., and Arsenault, M. (2009). Gene profiling of left ventricle eccentric hypertrophy in aortic regurgitation 
in rats: rationale for targeting the $\beta$-adrenergic and renin-angiotensin systems. American Journal of Physiology-Heart and Circulatory Physiology, 296(3):H669-H677.

Dent, M. R., Tappia, P. S., and Dhalla, N. S. (2010a). Gender differences in apoptotic signaling in heart failure due to volume overload. Apoptosis, 15(4):499-510.

Dent, M. R., Tappia, P. S., and Dhalla, N. S. (2010b). Gender differences in cardiac dysfunction and remodeling due to volume overload. J Card Fail, 16(5):439-49.

Deroo, B. J. and Korach, K. S. (2006). Estrogen receptors and human disease. The Journal of clinical investigation, 116(3):561-570.

Drolet, M. C., Lachance, D., Plante, E., Roussel, E., Couet, J., and Arsenault, M. (2006). Gender-related differences in left ventricular remodeling in chronic severe aortic valve regurgitation in rats. J Heart Valve Dis, 15(3):345-51.

Evangelista, A., Tornos, P., Sambola, A., Permanyer-Miralda, G., and Soler-Soler, J. (2005). Long-term vasodilator therapy in patients with severe aortic regurgitation. New England Journal of Medicine, 353(13):1342-1349.

Gardner, J. D., Brower, G. L., and Janicki, J. S. (2002). Gender differences in cardiac remodeling secondary to chronic volume overload. J Card Fail, 8(2):101-7.

Gardner, J. D., Murray, D. B., Voloshenyuk, T. G., Brower, G. L., Bradley, J. M., and Janicki, J. S. (2009). Estrogen attenuates chronic volume overload induced structural and functional remodeling in male rat hearts. American Journal of Physiology-Heart and Circulatory Physiology, 298(2):H497-H504.

Greenberg, B., Massie, B., Bristow, J. D., Cheitlin, M., Siemienczuk, D., Topic, N., Wilson, R., Szlachcic, J., and Thomas, D. (1988). Long-term vasodilator therapy of chronic aortic insufficiency. a randomized double-blinded, placebo-controlled clinical trial. Circulation, 78(1):92-103.

Iorga, A., Li, J., Sharma, S., Umar, S., Bopassa, J. C., Nadadur, R. D., Centala, A., Ren, S., Saito, T., Toro, L., Wang, Y., Stefani, E., and Eghbali, M. (2016). Rescue of pressure overload-induced heart failure by estrogen therapy. Journal of the American Heart Association, 5(1):e02482.

Iorga, A., Umar, S., Ruffenach, G., Aryan, L., Li, J., Sharma, S., Motayagheni, N., Nadadur, R. D., Bopassa, J. C., and Eghbali, M. (2018). Estrogen rescues heart failure through estrogen receptor beta activation. Biology of sex differences, 9(1):48.

Kararigas, G., Nguyen, B. T., and Jarry, H. (2014). Estrogen modulates cardiac growth through an estrogen receptor $\alpha$-dependent mechanism in healthy ovariectomized mice. Molecular and cellular endocrinology, 382(2):909-914.

Lachance, D., Dhahri, W., Drolet, M.-C., Roussel, É., Gascon, S., Sarrhini, O., Rousseau, J. A., Lecomte, R., Arsenault, M., and Couet, J. (2014). Endurance training or beta-blockade can partially block the energy metabolism remodeling taking place in experimental chronic left ventricle volume overload. BMC cardiovascular disorders, 14(1):190.

Lachance, D., Plante, E., Bouchard-Thomassin, A. A., Champetier, S., Roussel, E., Drolet, M. C., Arsenault, M., and Couet, J. (2009). Moderate exercise training improves survival and ventricular remodeling in an animal model of left ventricular volume overload. Circ Heart Fail, 2(5):437-45.

Leinwand, L. A. (2003). Sex is a potent modifier of the cardiovascular system. J Clin Invest, 112(3):302-7. Leon, M. B., Smith, C. R., Mack, M. J., Makkar, R. R., Svensson, L. G., Kodali, S. K., Thourani, V. H., Tuzcu, E. M., Miller, D. C., and Herrmann, H. C. f. t. P. . I. (2016). Transcatheter or surgical aortic-valve replacement in intermediate-risk patients. New England Journal of Medicine, 374(17):1609-1620.

Lin, M., Chiang, H.-T., Lin, S.-L., Chang, M.-S., Chiang, B. N., Kuo, H.-W., and Cheitlin, M. D. (1994). Vasodilator therapy in chronic asymptomatic aortic regurgitation: enalapril versus hydralazine therapy. Journal of the American College of Cardiology, 24(4):1046-1053.

Mahmoodzadeh, S. and Dworatzek, E. (2019). The role of $17 \beta$-estradiol and estrogen receptors in regulation of ca2+ channels and mitochondrial function in cardiomyocytes. Frontiers in endocrinology, $10: 310$.

Maric-Bilkan, C., Arnold, A. P., Taylor, D. A., Dwinell, M., Howlett, S. E., Wenger, N., Reckelhoff, J. F., Sandberg, K., Churchill, G., Levin, E., and Lundberg, M. S. (2016). Report of the national heart, lung, and blood institute working group on sex differences research in cardiovascular disease: scientific questions and challenges. Hypertension, 67(5):802-807.

Murphy, E. (2011). Estrogen signaling and cardiovascular disease. Circulation research, 109(6):687-696.

Plante, E., Couet, J., Gaudreau, M., Dumas, M.-P., Drolet, M.-C., and Arsenault, M. (2003). Left ventricular response to sustained volume overload from chronic aortic valve regurgitation in rats. 
Journal of cardiac failure, 9(2):128-140.

Plante, E., Lachance, D., Champetier, S., Drolet, M. C., Roussel, E., Arsenault, M., and Couet, J. (2008). Benefits of long-term beta-blockade in experimental chronic aortic regurgitation. Am J Physiol Heart Circ Physiol, 294(4):H1888-95.

Rajabi, M., Kassiotis, C., Razeghi, P., and Taegtmeyer, H. (2007). Return to the fetal gene program protects the stressed heart: a strong hypothesis. Heart failure reviews, 12(3-4):331-343.

Singh, J. P., Evans, J. C., Levy, D., Larson, M. G., Freed, L. A., Fuller, D. L., Lehman, B., and Benjamin, E. J. (1999). Prevalence and clinical determinants of mitral, tricuspid, and aortic regurgitation (the framingham heart study). The American journal of cardiology, 83(6):897-902.

Taegtmeyer, H., Sen, S., and Vela, D. (2010). Return to the fetal gene program. Annals of the New York Academy of Sciences, 1188(1):191-198.

Zühlke, L. J., Beaton, A., Engel, M. E., Hugo-Hamman, C. T., Karthikeyan, G., Katzenellenbogen, J. M., Ntusi, N., Ralph, A. P., Saxena, A., Smeesters, P. R., Watkins, D., Zilla, P., and Carapetis, J. (2017). Group a streptococcus, acute rheumatic fever and rheumatic heart disease: epidemiology and clinical considerations. Current treatment options in cardiovascular medicine, 19(2):15. 

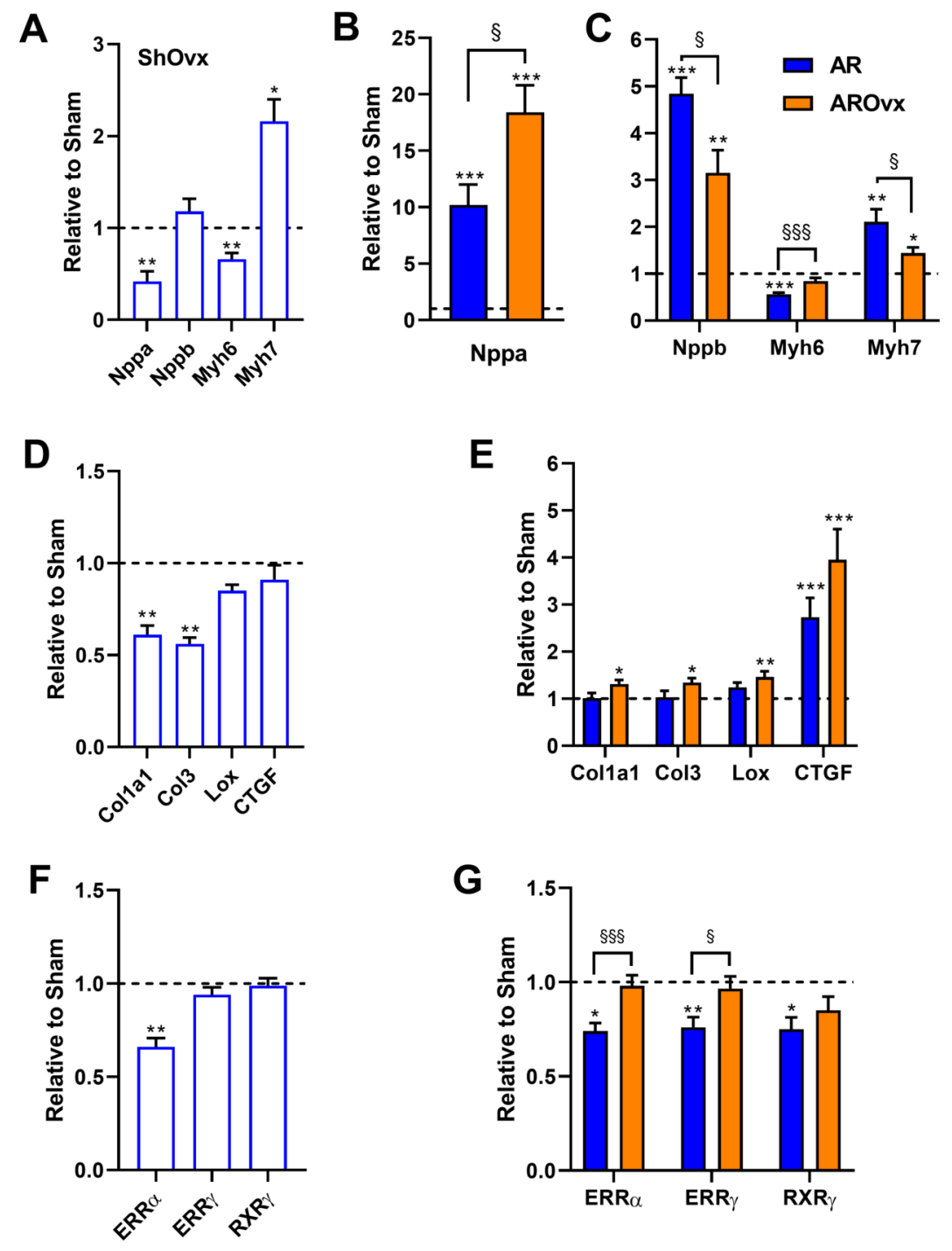

Figure 3. Evaluation by real-time quantitative RT-PCR of LV mRNA levels of genes encoding for hypertrophy markers (A, B and C), extracellular matrix genes (D and E) and transcription factors implicated in the control of myocardial energetics ( $F$ and $G)$. The results are reported as the mean +/SEM ( $n=6 /$ gr.) relative to non-Ovx Sham group (Panels A, D and F) or to respective Sham group (non-Ovx (Blue) or Ovx (Orange) (Panels B, C, E and G). Messenger RNA levels of the respective sham group were normalized to 1 and are represented by the dotted line. $*: \mathrm{p}<0.05, * *: \mathrm{p}<0.01$ and $* * *: \mathrm{p}$ $<0.001$ vs. respective sham group. $\S: \mathrm{p}<0.05$ and $\S \S \S: \mathrm{p}<0.001$ between indicated groups. 

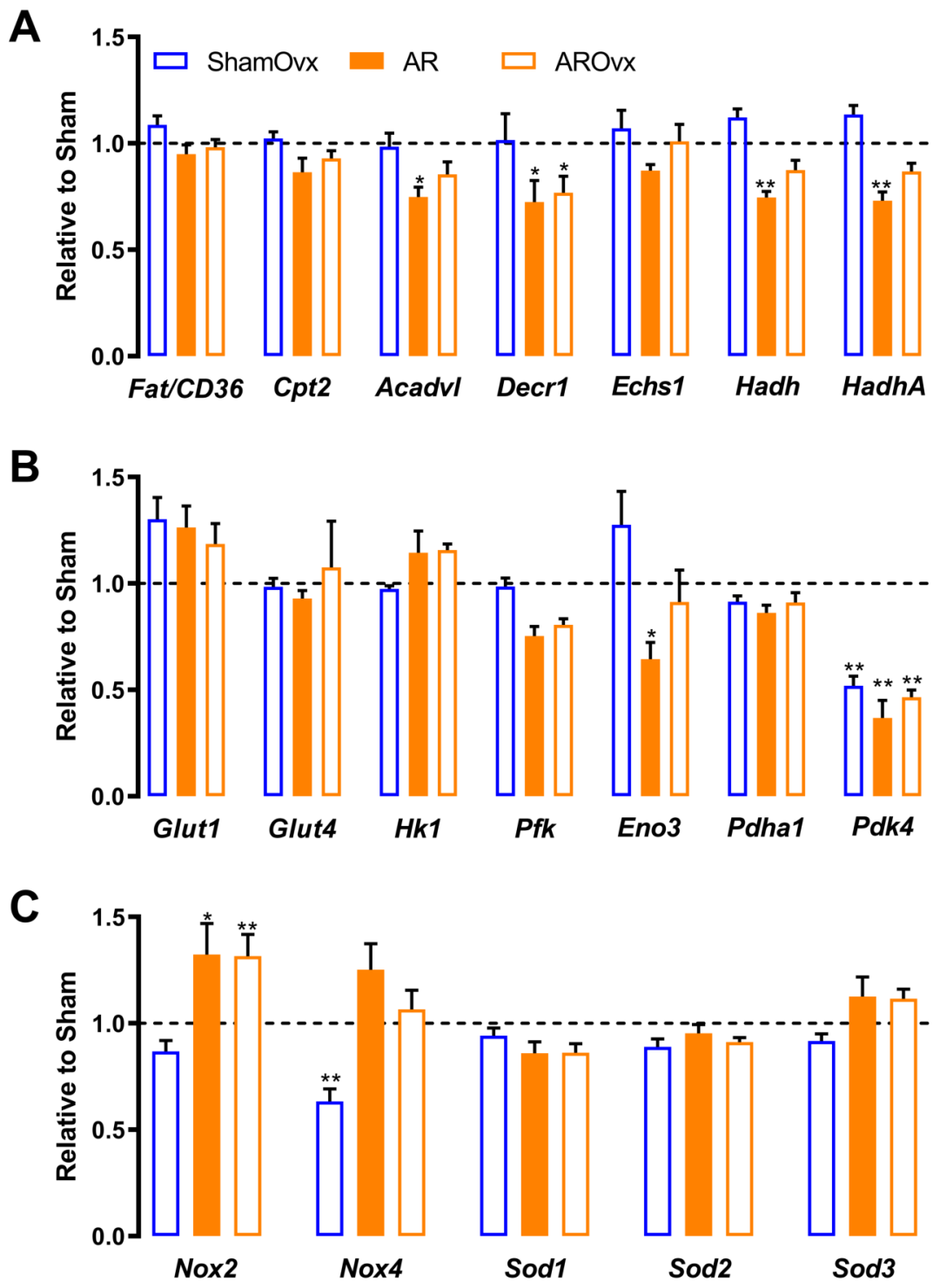

Figure 4. Genes implicated in energetics (panels A and B) and reactive oxygen species metabolism (C) are not modulated by the loss of estrogens in ShamOvx and AROvx rats. The results are reported as the mean + /- SEM ( $n=6 /$ gr.) relative to non-Ovx Sham group. Messenger RNA levels of non-Ovx Sham group were normalized to 1 and are represented by the dotted line. *: $\mathrm{p}<0.05$ and **: $\mathrm{p}<0.01$ vs. non-Ovx sham group. 
A

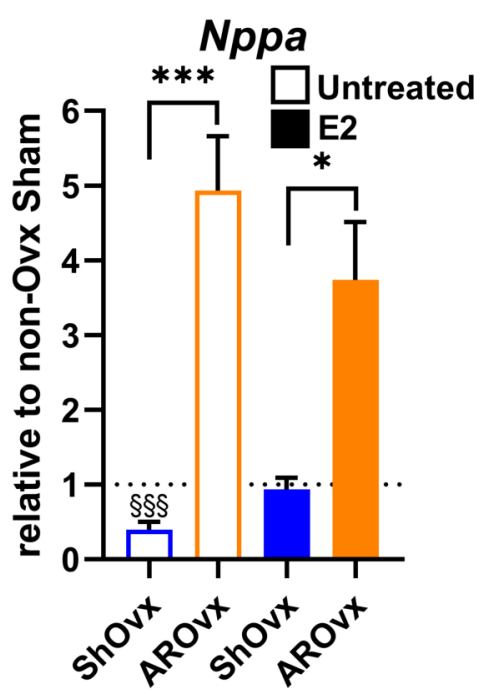

C

Myh6

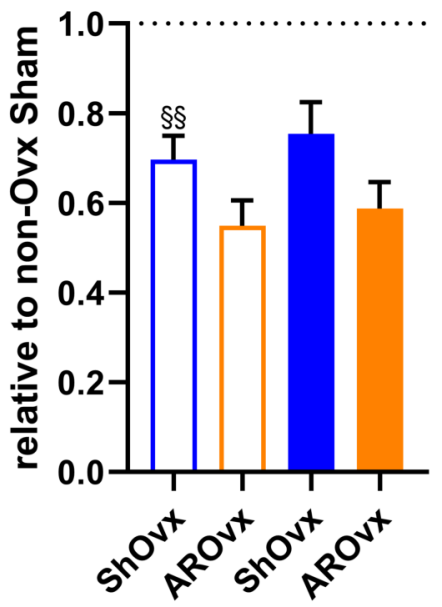

B

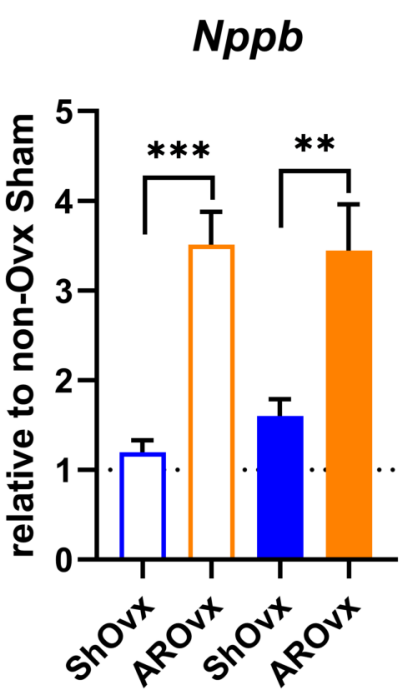

D

Myh7

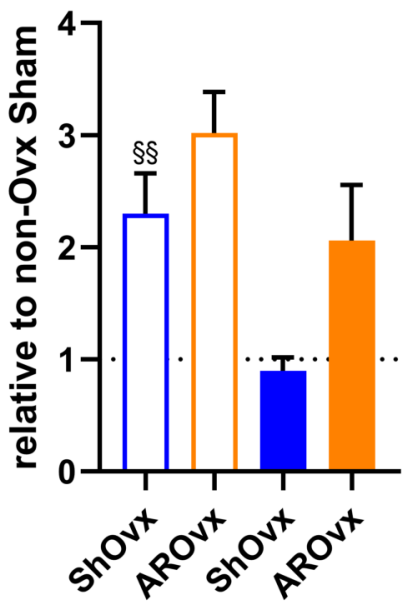

Figure 5. Evaluation by real-time quantitative RT-PCR of LV mRNA levels of genes encoding for hypertrophy markers in Sham Ovx and AR Ovx rats receiving (orange) or not (blue) 17beta-estradiol (E2) replacement. A: Nppa, B: Nppb, C: Myh6 and D: Myh7. The results are reported as the mean +/- SEM $(\mathrm{n}=6 /$ gr.) relative to non-Ovx Sham group (set to 1; dotted line). $*: \mathrm{p}<0.05$, **: $\mathrm{p}<0.01$ and between $* * *: \mathrm{p}<0.001$ indicated groups. $\S \S: \mathrm{p}<0.01$ and $\S \S \S: \mathrm{p}<0.001$ vs. non-ovx Sham group. 